ARTICLE

Received 1 Apr 2015 | Accepted 7 Jul 2015 | Published 24 Aug 2015

DOI: $10.1038 /$ ncomms 9011

OPEN

\title{
A chameleon-inspired stretchable electronic skin with interactive colour changing controlled by tactile sensing
}

Ho-Hsiu Chou', Amanda Nguyen², Alex Chortos ${ }^{3}$, John W.F. To ${ }^{1}$, Chien Lu', Jianguo Mei ${ }^{1}$, Tadanori Kurosawa', Won-Gyu Bae', Jeffrey B.-H. Tok ${ }^{1} \&$ Zhenan Bao,3

Some animals, such as the chameleon and cephalopod, have the remarkable capability to change their skin colour. This unique characteristic has long inspired scientists to develop materials and devices to mimic such a function. However, it requires the complex integration of stretchability, colour-changing and tactile sensing. Here we show an all-solution processed chameleon-inspired stretchable electronic skin (e-skin), in which the e-skin colour can easily be controlled through varying the applied pressure along with the applied pressure duration. As such, the e-skin's colour change can also be in turn utilized to distinguish the pressure applied. The integration of the stretchable, highly tunable resistive pressure sensor and the fully stretchable organic electrochromic device enables the demonstration of a stretchable electrochromically active e-skin with tactile-sensing control. This system will have wide range applications such as interactive wearable devices, artificial prosthetics and smart robots.

\footnotetext{
${ }^{1}$ Department of Chemical Engineering, Stanford University, Stanford, California 94305, USA. ${ }^{2}$ Department of Electrical Engineering, Stanford University, Stanford, California 94305, USA. ${ }^{3}$ Materials Science and Engineering, Stanford University, Stanford, California 94305, USA. Correspondence and requests for materials should be addressed to Z.B. (email: zbao@stanford.edu).
} 
$\mathrm{H}$ uman skin provides a remarkable network of sensors with highly sensitive pressure, temperature and vibration sensing ${ }^{1-3}$. Skin can transduce environmental stimuli into physiological signals, which are then interpreted by brain. Electronic skin (e-skin) is an artificial skin that mimics the properties of skin using electronic devices. Inspired by human skin, e-skin has been found many potential applications ${ }^{4-25}$, such as wearable devices, artificial prosthetics, health monitoring and smart robots.

Unlike human skin, both animal and insect skin exhibit additional functions, for example, the chameleon's skin has colour-changing abilities. A chameleon shifts its skin colour through controlling the skin pigment cell for purposes in camouflage ${ }^{26}$, temperature maintenance and communication. Since chameleons cannot generate any body heat, the colour of their skin can in turn be used to regulate their body temperature. Mimicking the colour-changing ability of chameleons can also be achieved using approaches such as mechanical or electrical control $^{27-31}$. Whitesides and colleagues ${ }^{31}$ reported a soft machine equipped with microfluidic channels that can be colour-filled or colour-flushed by pumping a coloured liquid through the channels. Rogers and colleagues ${ }^{32}$ reported an adaptive optoelectronic camouflage system using a leucodye composite, which can produce black and white patterns to match its surroundings. Zhao and colleagues ${ }^{33}$ reported a soft material system for generating voltage-controlled on-demand fluorescent patterns that can be modulated to exhibit manifold geometries. Despite these achievements, the above devices nonetheless only demonstrated colour-changing abilities, it lacks the crucial function of skin, namely, tactile sensing. Javey and colleagues ${ }^{13}$ recently furthered these advancements by describing a userinteractive e-skin for instantaneous pressure visualization on a polyimide substrate. This e-skin is capable of correlating the applied pressure to the brightness of the devices as well as spatially mapping the applied pressure. However, the e-skin needs a constant bias to maintain its light/colour, which is not ideal for low power consumption system. In addition, since the device is fabricated on a plastic substrate, it is not stretchable.

Human skin is generally considered as an 'ideal' low power consumption sensor. To mimic this property, creating a low power consumption system is highly relevant for e-skin applications. In addition, presence of both parameters in tactile sensing and stretchability are also important because tactile sensing of skin allows the body to communicate with the outside environment, whereas the stretchability of skin enables us free movement.
In the following, we describe a bio-inspired stretchable e-skin with interactive colour-changing and tactile-sensing properties (Fig. 1). This concept is realized through the development and integration of a stretchable highly tunable resistive pressure sensor (PS) and stretchable organic electrochromic devices (ECDs). This e-skin, besides detecting applied pressure, is also able to distinguish varying applied pressures through real-time visible colour change. This work demonstrates low power consumption, interactive and colour-changeable e-skin, which is readily prepared by a cost-efficient all-solution processing approach.

\section{Results}

Stretchable, transparent and highly tunable resistive PSs. Pressure sensing is one of the key functions of e-skin devices. Development of PSs that can mimic the pressure-sensing properties of natural skin requires proper design of both materials and devices ${ }^{6,34-39}$. In our previous study, we have developed a highly sensitive flexible PS with microstructured polydimethylsiloxane (PDMS) dielectric layer6. Various other PSs with high pressure sensitivity have been reported recently ${ }^{5-7,39,40}$ While high sensitivity is a crucial characteristic of PSs, facile implementation in real-world applications also necessitates tunable response sensitivities and tunable threshold of resistance switching range to meet different requirements. To integrate e-skin with a stretchable organic ECD, a stretchable and highly tunable resistive PS is needed. In addition, since electrochromic colour is voltage modulated, the pressuresensitive element therefore needs to modify its voltage upon applied pressure. A comparator circuit is the most straightforward and simplest approach to implement the above requirement.

Even though many resistive PSs have been reported, their resistance value and threshold of resistance switching ranges are not easy to adjust. Furthermore, the change in resistance value is insufficient to be switched from non-conductive to highly conductive. To meet these requirements, we here developed a new type of stretchable, transparent and highly tunable resistive PS based on an elastic pyramidal-microstructured PDMS with a layer of spray-coated single-wall carbon nanotubes (SWNTs). The PS is uniquely designed to be capable of adjusting the height of the SWNTs on the pyramid, and also in controlling the resistance response and the threshold of resistance switching range.

The schematic process for the fabrication of the PS is shown in Fig. 2a. First, we used a pyramidal-microstructured PDMS with a

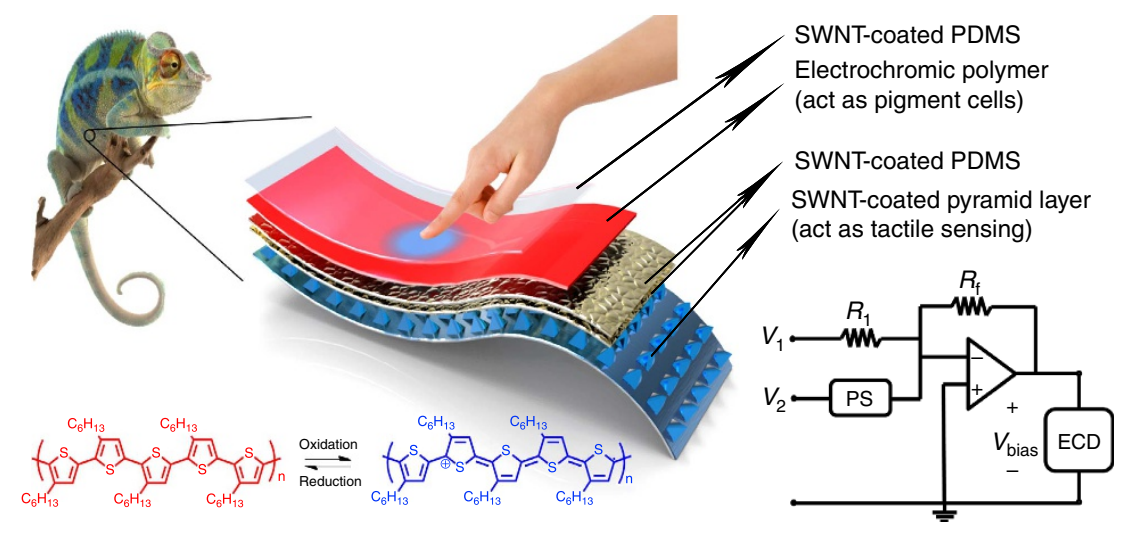

Figure 1 | Illustration of the concept of a chameleon-inspired e-skin. Also shown are (bottom left) the structures of both the neutral and oxidized states of the electrochromic polymer in poly(3-hexylthiophene-2,5-diyl, P3HT), and (bottom right) a schematic of the circuit layout (PS, pressure sensor; ECD, electrochromic device). (Ambanja panther chameleon and young hand images from www.123rf.com). 
a
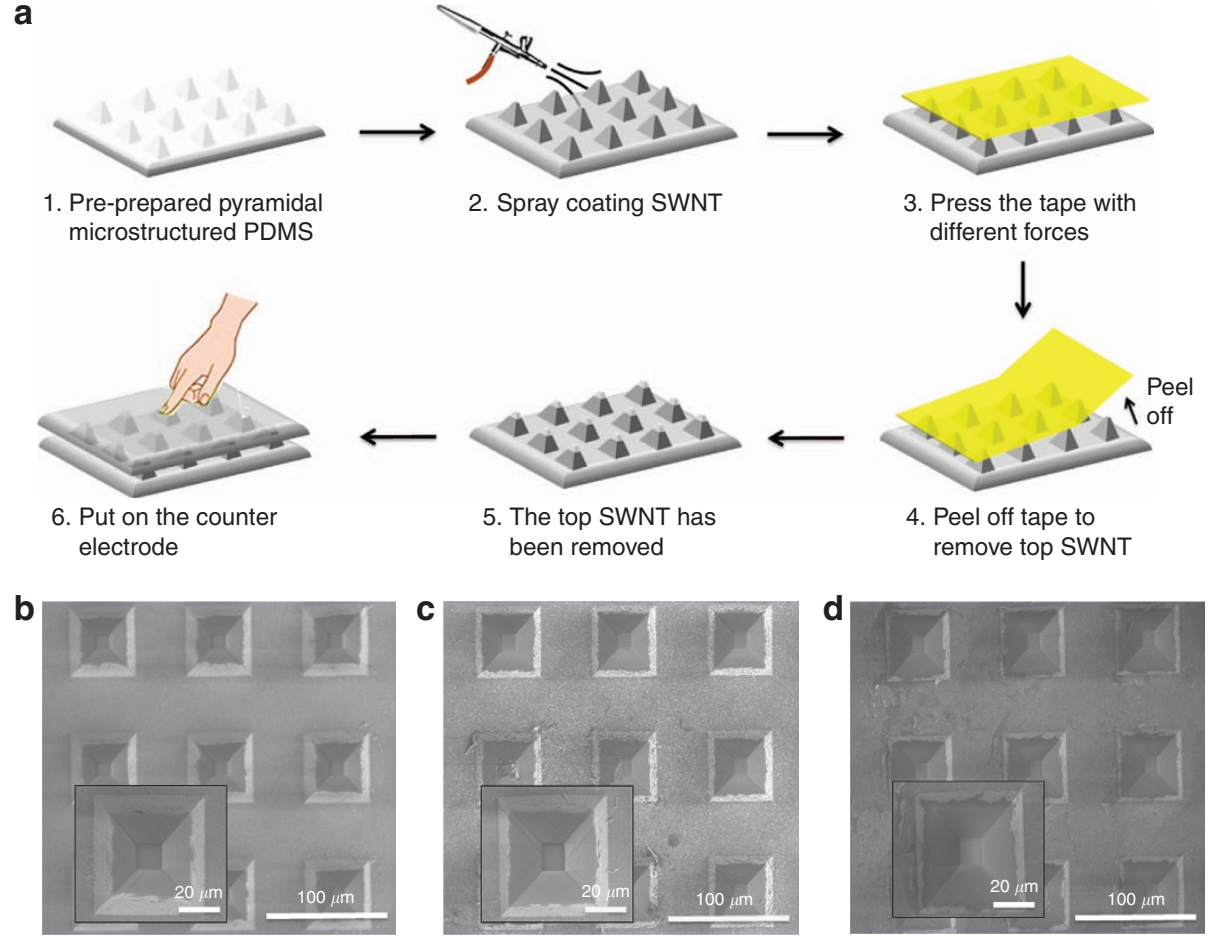

Figure 2 | A schematic of the fabrication process and their SEM images. (a) A schematic for the fabrication of PSs PS-1, PS-10 and PS-30. (b) The SEM image of PS-1. (c) The SEM image of PS-10. (d) The SEM image of PS-30. Higher magnification SEM images of individual pyramid are provided in the inset.

$34 \mu \mathrm{m}$ in height, $10 \times 10 \mu \mathrm{m}^{2}$ for the top, $50 \times 50 \mu \mathrm{m}^{2}$ for the base and $41 \mu \mathrm{m}$ periodic spacing. It was fabricated using our previously described methods (Supplementary Fig. 1a) ${ }^{6,41}$. Second, the SWNT was uniformly spray coated on top of the pyramidal-microstructured PDMS surface. After the SWNT spray coating, we observed that the $10 \times 10 \mu \mathrm{m}^{2}$ top of pyramids is covered by SWNT, leading to low resistance even without any applied pressure (Supplementary Fig. 1b). Therefore, we used a kapton tape to remove the SWNT from the top of the pyramid. A key aspect of our approach is that we hypothesized that we can easily apply different pressures (that is, 1,10 and $30 \mathrm{kPa}$ ) on the kapton tape to adjust the height of the SWNT on the pyramidalmicrostructured PDMS. Hence, we have accordingly designated these sensors as PS-1, PS-10 and PS-30, respectively. Notably, pyramids with heights of $34 \mu \mathrm{m}$ were used in this study because it can provide a larger dynamic range for modulating the height of SWNTs on the pyramids compared with a smaller height ${ }^{6,17,42,43}$. Indeed, it was observed that the larger the pressure applied on the tape, the less SWNT remained on the pyramids (Fig. 2b-d). This provided both the necessary tunable resistance and tunable threshold of resistance switching range for the PS. Finally, a counter electrode composed of a layer of SWNTs on a flat surface of PDMS was placed on the top of the SWNTs partially coated pyramids.

Figure $3 a-c$ shows the pressure response for four consecutive measurements (that is, press, blue line; release, red line) of the pyramidal-microstructured PDMS corresponding to PS-1, PS-10 and PS-30, respectively. The resulting square pressure-sensitive pad was $1 \mathrm{~cm}^{2}$ in size. Slightly higher resistances were observed with PS-1 and PS-10 for the first cycle, the subsequent cycles were reproducible. Based on our design, when no pressure is applied, the sensor is non-conductive and should prevent the current flowing due to no SWNTs coating on the top of the pyramid. It becomes conductive and the resistance drops with an applied pressure. As expected, PS-1 showed the lowest resistance at the same applied pressure, which is a result of the higher coverage of SWNTs for PS-1. All the PSs PS-1, PS-10 and PS-30 exhibited changes in resistance of up to five orders of magnitude by applying pressure between 0 and $20 \mathrm{kPa}, 0$ and $50 \mathrm{kPa}$, and 0 and $165 \mathrm{kPa}$, respectively. In addition, Fig. $3 \mathrm{~d}$ shows that the threshold of the resistance switching of PS-1, PS-10 and PS-30 was gradually increased as a function of the height of the SWNT, showing onset detection of pressures at $2.2,7.4$ and $13.3 \mathrm{kPa}$, respectively (second forward). This demonstrates that the height of the SWNTs can be related to the threshold of the resistance switching range, which is defined as the point of the resistance of the PS in which it begins to drop markedly. Our obtained results indicated that both the resistance range and the threshold of resistance switching are highly tunable through simply modifying the height of the SWNT structures. Here we target pressure range equivalent to human touch. Normal grip forces to hold objects are in the range of several $\mathrm{kPa}$ to several hundred $\mathrm{kPa}$. The pressure applied during a handshake is in the order of several tens of $\mathrm{kPa}$. Therefore, the easy and tunable design of our PS is ideal for human interactive system, which will be demonstrated in the integration section.

To test the stretchability of our sensors, we stretched PS-10 at both 20 and $50 \%$ strains. The PS still maintained its functionality, as shown in Fig. 3e. The PS-10 exhibited changes in resistance of up to five orders of magnitude at $20 \%$ strain. Even at $50 \%$ strain, it can still maintain the changes in resistance of up to four orders of magnitude. As a result, we have demonstrated stretchable and transparent resistive PSs, with the advantage of having a widely tunable dynamic range by using a simple process. To further access the resistance response, we analyse the slopes of response curve of PS. As shown in Fig. 3f, five different kinds of slopes are observed in the response curve of PS-10. As the pressure is applied, slope 1 corresponds to the counter electrode touching the non-SWNT-coated pyramid tip, but before the counter electrode touches the SWNT-coated part. During this period, we observed 
a
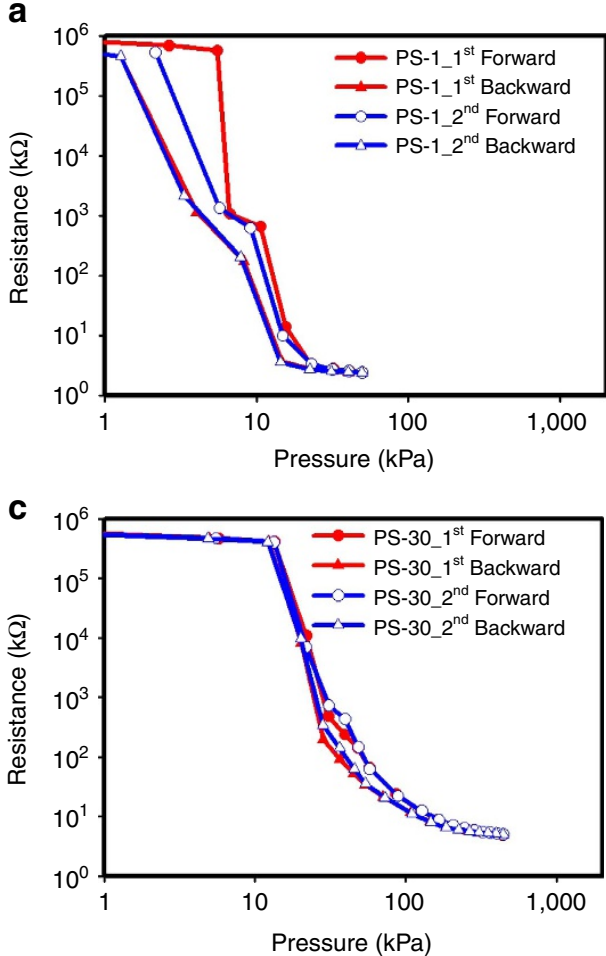

e

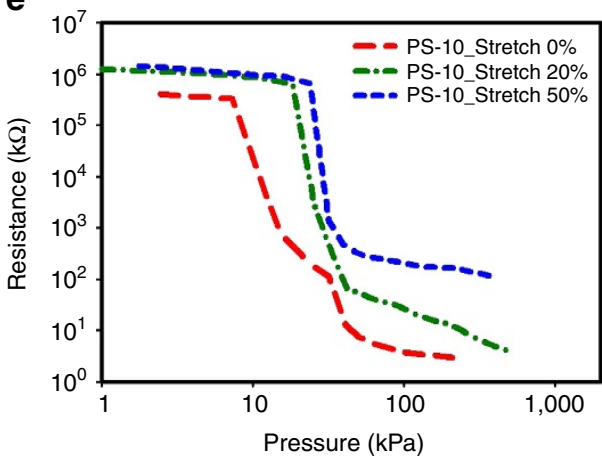

b

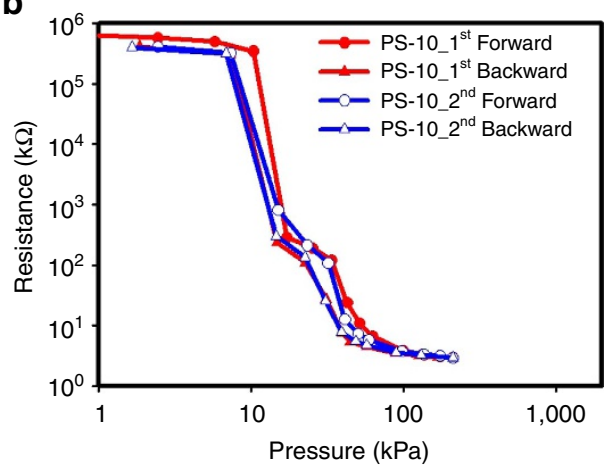

d 100

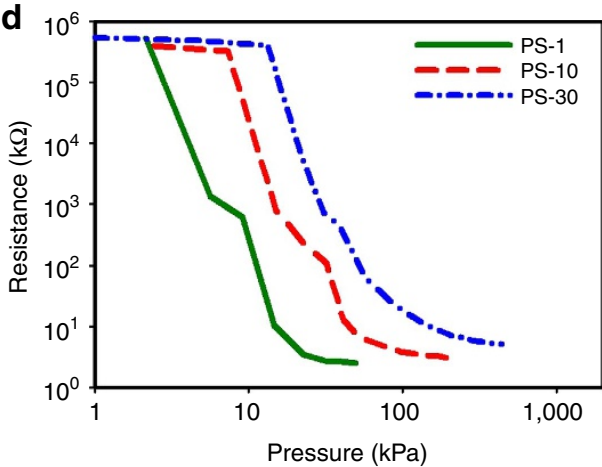

f

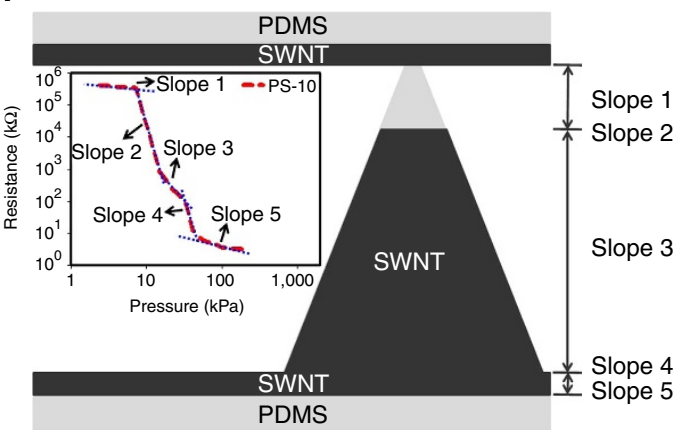

Figure 3 | Pressure-sensing characterization of PS. The pressure response for four consecutive measurements of (a) PS-1, (b) PS-10 and (c) PS-30. (d) The comparison of the resistance response and the threshold of resistance switching range of PSs (second forward). (e) The comparison of the resistance response of PS-10 at different strains. (f) A schematic diagram of single SWNT-coated pyramidal-microstructured PDMS (inset: each slope of the resistance response of PS-10).

that the slope is nearly flat and this is because the PS is maintained in a non-conductive status. Slope 2 is when the counter electrode first comes into contact with the SWNT-coated parts of the pyramids. A sharp slope is observed in this period, since the SWNT-coated part of the pyramids provide a large sheet resistance to reduce the resistance markedly. Slope 3 is generated when the counter electrode is in contact with the SWNT-coated pyramid just before touching the base of SWNT-coated PDMS. Slope 4 is generated when the counter electrode touches the base of SWNT-coated PDMS, this is referred to as. Finally, the region where the curve of the resistance response of PS-10 becomes saturated is slope 5 .

To illustrate the stability of our fabricated PS, we measured multiple cyclic data of PS-10. As shown in Supplementary Fig. 2, the resistance of the PS remained consistent at both $0 \mathrm{kPa}$ $\left(\sim 2 \times 10^{6} \mathrm{k} \Omega\right)$ and $22 \mathrm{kPa}(\sim 50 \mathrm{k} \Omega)$ after over 300 cycles. This observation illustrates that our resistive PS has both long-term stability and reliability as the SWNTs remained well attached on the surface of pyramidal PDMS structures. In addition, we have fabricated a $3 \times 3 \mathrm{~cm}^{2}$ PS to demonstrate that the pyramidal structure and the SWNT removal processes for the PSs can potentially be applied to large area devices (Supplementary Fig. 3). With this PS, we observed excellent resistance response curves and reproducibility after a $10 \times$ repeated run. Furthermore, the change in resistance is $\sim 5$ orders of magnitude, which demonstrates that the PS can indeed be applied over a large area for practical artificial skin applications.

Fully stretchable organic ECDs. To fabricate the stretchable interactive colour-changeable e-skin, a stretchable ECD is selected to integrate with the highly tunable resistive PS due to its ease in fabrication, multi- and tunable colours. In addition, human skin is an ideal low power consumption sensor. To mimic this property, reducing the power consumption is essential for practical e-skin applications. The ECD has the advantage of colour retention being called the 'colour memory effect' and has also been demonstrated to be low power consuming ${ }^{4-46}$. Therefore, the development of stretchable ECD for e-skin applications is ideal to achieve both the concept of low power consumption and 
colour-changeable properties. In a previous report on stretchable ECD by Lee and coworkers ${ }^{47}$, they used an inorganic electrochromic material $\mathrm{WO}_{3}$ for colour switching. However, a liquid electrolyte was used that had the potential issue of liquid leakage, and will lead to a critical weakness for e-skins when exposed to a variety of wear-and-tear forces. In addition, they used a rigid platinum wire as the counter electrode, which is not stretchable, and a thick silver nanowire film is used as the nontransparent electrode, which will be difficult to implement in the multiplex ECDs and incompatible with dual-electrochromic layer. Consequently, such an ECD is not capable of stretching the counter electrode and electrolyte, and is not suitable for making the fully stretchable colour-changeable e-skin. We choose polymer-based ECD owing to their ease of colour tuning through molecular design ${ }^{9,48,49}$, good stretchability 8,50 and promising application in printed devices ${ }^{48,51,52}$, thereby producing a mechanically robust and scalable ECD to overcome present obstacles. Here the stretchable polymer ECDs are designed and fabricated to be fully stretchable, including the counter electrode and electrolyte. Our ECD is fabricated on an elastic and biocompatible PDMS substrate. Thin films of SWNTs were used as transparent electrodes because of their high stretchability and good conductivity. They were coated by spray coating on the top of the PDMS substrate 4 . Various electrochromic (EC) polymers have previously reported ${ }^{53-58}$. Poly(3-hexylthiophene-2,5-diyl) (P3HT) was used as an electrochromic layer for demonstration of concept due to its good stretchability ${ }^{59-62}$. It exhibits a dark red colour in its neutral form and pale blue colour in its oxidized form.

Figure 4a shows the ultraviolet-visible spectra of the stretchable polymer-based ECDs. The maximum absorption of the neutral P3HT was ca. $550 \mathrm{~nm}$, whereas the oxidized P3HT was redshifted to ca. $800 \mathrm{~nm}$. The kinetic absorption measurement is used to determine the switching behaviour and stability of the stretchable ECDs at $550 \mathrm{~nm}$ through cycling the bias voltage between 1.0 and $-1.0 \mathrm{~V}$ with a $10-\mathrm{s}$ step, as shown in Fig. $4 \mathrm{~b}$. Notably, its low driving voltage of $\pm 1.0 \mathrm{~V}$ is beneficial for the concept of low power consumption e-skin. At $0 \%$ strain, the switching time for neutral state (turning dark red) and oxidized state (turning pale blue) was 1.4 and $1.2 \mathrm{~s}$, respectively (Fig. 4c). At $20 \%$ strain, the switching time for neutral state (turning dark red) and oxidized state (turning pale blue) was only slightly
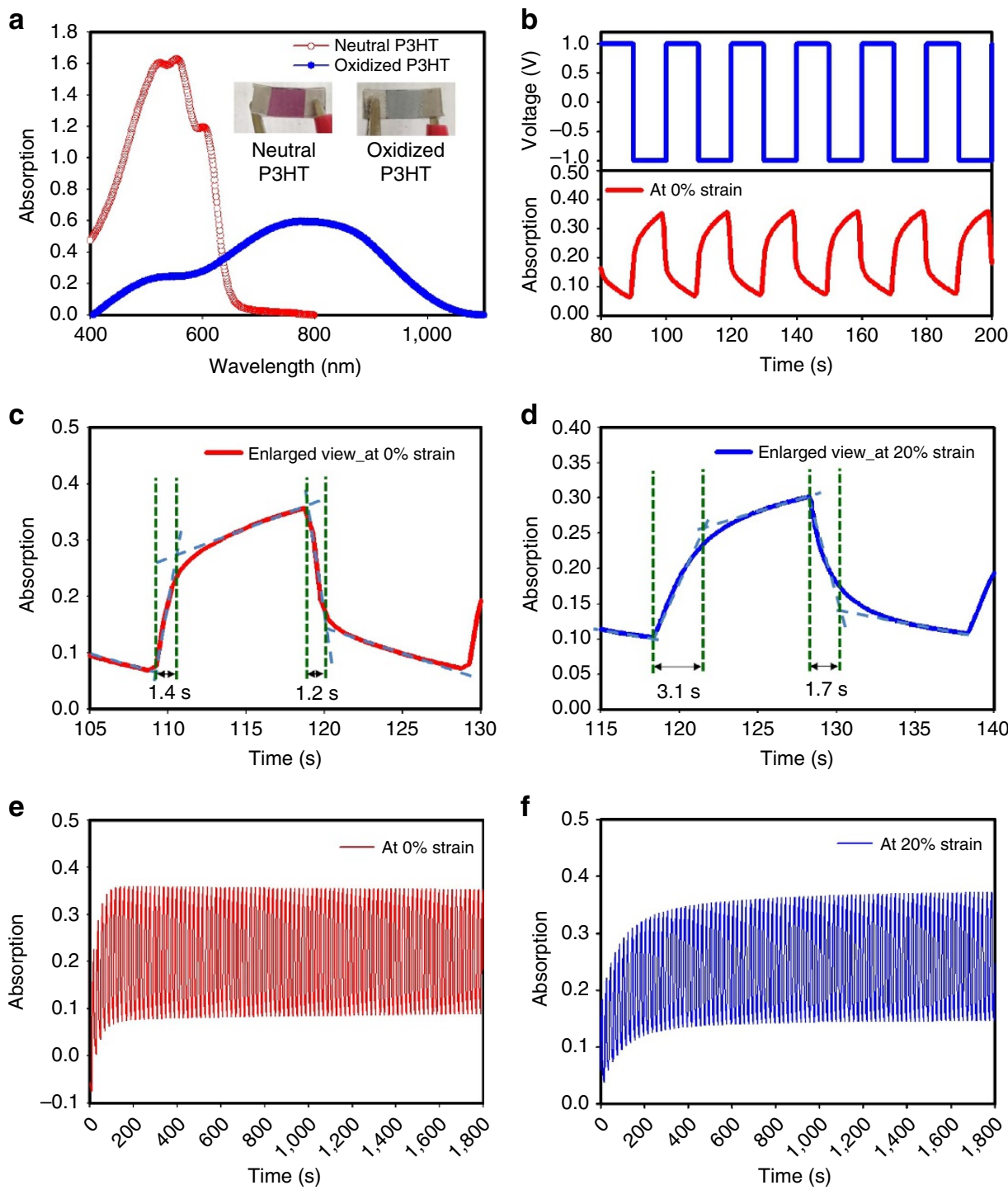

Figure 4 | The properties of the stretchable polymer-based ECDs. (a) The ultraviolet-visible spectra of the neutral and oxidized P3HT. (b) The colour switching behaviour of the ECDs at $0 \%$ strain. (c) The enlarged spectra of the single switching cycle at $0 \%$ strain. (d) The enlarged spectra of the single switching cycle at $20 \%$ strain. (e) The cyclic switching at $0 \%$ strain. (f) The cyclic switching at $20 \%$ strain. 
increased at 3.1 and $1.7 \mathrm{~s}$, respectively (Fig. 4d). Both the stretchable organic ECDs at both 0 and $20 \%$ strains showed stable cyclic switching, and the colour contrast was maintained at over $90 \%$ even after long-term measurement (Fig. 4e,f). In addition, the ECDs at $50 \%$ strain showed the function of the switching cycle (Supplementary Fig. 4). However, the switching speed was degraded because of the increased resistance. Notably, we chose to use commercially available $\mathrm{P} 3 \mathrm{HT}$ to demonstrate the fully stretchable ECDs. We believe that improvements of device performance can be further achieved by design and synthesis of improved electrochromic polymers.

To elucidate the relationship between strain and electrical properties of the neutral and oxidized P3HT/SWNT stack in ECDs, we measured the resistance versus strain for both the neutral and oxidized P3HT/SWNT films. The films were first both soaked in the liquid electrolyte $\left(0.1 \mathrm{M} \mathrm{LiClO}_{4}\right.$ in $\left.\mathrm{CH}_{3} \mathrm{CN}\right)$ for $30 \mathrm{~s}$ before the stretching measurement. Figure $5 \mathrm{a}$ plots the normalized resistance of these two thin films when stretched from 0 to $100 \%$ strain. The resistance of the oxidized P3HT at $100 \%$ strain increased only $\sim 1.9$ times, whereas the neutral P3HT was increased to $\sim 3.2$ times at $100 \%$ strain. We can observe that at $20 \%$ strain, the resistance of the oxidized and neutral P3HT was only increased 1.23 and 1.12 times compared to its original resistance, respectively. If we stretch the thin films to $50 \%$ strain, the resistance of the neutral and oxidized $\mathrm{P} 3 \mathrm{HT}$ was increased to 1.85 and 1.33 times, respectively. We speculated that the much higher increase in the resistance of neutral P3HT led to the degradation of the switching speed of ECD at 50\% stain. The slower resistance increases in the oxidized P3HT thin film may be because of the higher concentrations of holes in the oxidized P3HT thin film, helping to maintain more conductive pathways even at elevated strains to inhibit the resistance increase. In addition, to further investigate the effects of strain on the ECDs, we compared the absorption spectra of P3HT under different strain levels (Fig. 5b). The absorption shoulder of P3HT at $600 \mathrm{~nm}$, typically assigned to the aggregation state of P3HT, increased when subjected to $>50 \%$ strain. This result suggests that the fraction of aggregated polymer increased during stretching, most likely due to strain-induced chain extension and subsequent crystallization.

A key parameter for wearable electronics is its weight. To demonstrate the lightweight capability, we used an ultrathin $1.2-\mu \mathrm{m}$-thick polyethylene naphthalate (PEN) film to fabricate the organic ECDs. In addition, the ultrathin PEN or polyethylene terephthalate has exhibited the capability of geometric stretchability $^{11}$. The fabrication process is similar to that of organic ECDs on PDMS substrate. The device weighed only $9.3 \mathrm{mg} \mathrm{cm}^{-2}$, which is lighter than the weight of a similarly sized device on textile, such as a conventional labcoat made from cotton polyester blend ( $16.7 \mathrm{mg} \mathrm{cm}^{-2}$; Supplementary Fig. 5).
These results indicate that organic ECDs are potentially promising for wearable devices. As a result, we have demonstrated that our stretchable organic ECDs have fast colour responses, are transparent and lightweight.

Chameleon-inspired stretchable e-skin integration. To produce a prototype, an integrated stretchable PS and stretchable ECD, denoted as 'PSEC', is fabricated. The schematic layout of the integrated system and its schematic circuitry is shown in Fig. 6a. Based on this integration, we can control the colour by simply applying various pressure levels. Figure $6 \mathrm{~b}$ shows the spectroscopic change measured by ultraviolet-visible absorption spectrum by applying different pressures from 0 to $200 \mathrm{kPa}$. Each of the applied pressure was first maintained for $10 \mathrm{~s}$, and the device was then turned off and the absorption spectrum was subsequently measured. We observed an absorption peak at $800 \mathrm{~nm}$ for the oxidized $\mathrm{P} 3 \mathrm{HT}$ before any pressure was applied. With increasing pressure, we observed the appearance of another absorption peak at $550 \mathrm{~nm}$ (which we attribute it due to the formation of neutral P3HT), and its intensity was observed to increase with increasing applied pressure. Various absorption spectrum/colours were used to further quantify the corresponding magnitude of the applied pressure. Through introducing various colours of the electrochromic polymers, the PSEC system could potentially be designed with a wide range of colours and, more importantly, be modulated by various pressures. As such, we will then be able to distinguish the different applied pressure level via the skin colours. In addition, to realize the power consumption property of the PSEC system, we measured if the absorption band/colour of the ECD at either natural or oxidized states. As Fig. 6c shown, both the absorption band/colour of neutral and oxidized P3HT can be maintained for $6 \mathrm{~h}$ at ambient environment without applying any additional bias. We only observed a slight increase in the absorption band of the ECDs after 1 day. This demonstrates that our colour-changeable e-skin does not require a constant bias to maintain the colour, which is important for the concept of a low power consumption e-skin. We also note that commercial electrochromic polymer $\mathrm{P} 3 \mathrm{HT}$ was used to produce the prototype. Hence, further improvement can be achieved by using the modified electrochromic polymers or better encapsulated processing.

We further investigated the response time of colour changes as a function of applied pressure using ultraviolet-visible measurements. We applied various pressures to the PSEC and maintained each pressure for $10 \mathrm{~s}$. Figure $6 \mathrm{~d}$ shows that the largest changing response of absorption was obtained using the highest pressure of $200 \mathrm{kPa}$. We then decrease the applied pressure down to 150,100 , 70,50 and $10 \mathrm{kPa}$, and the corresponding changes in absorption were observed. We observed that the various applied pressures
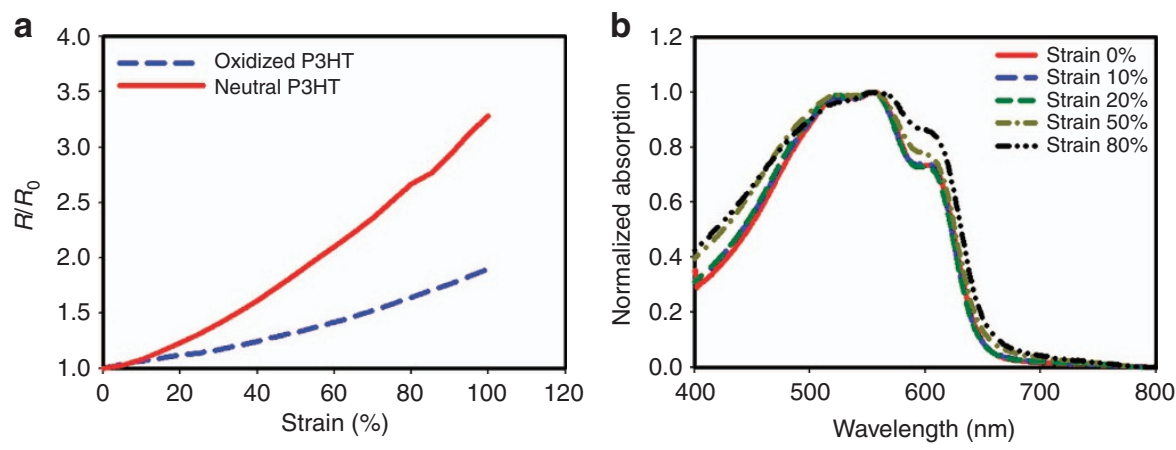

Figure 5 | Mechanical and absorption characteraization of ECDs. (a) The resistance comparsion of neutral and oxidized P3HT for strains ranging from 0 to $100 \%$. (b) The absorption spectra of P3HT ECD at different strains. 
a
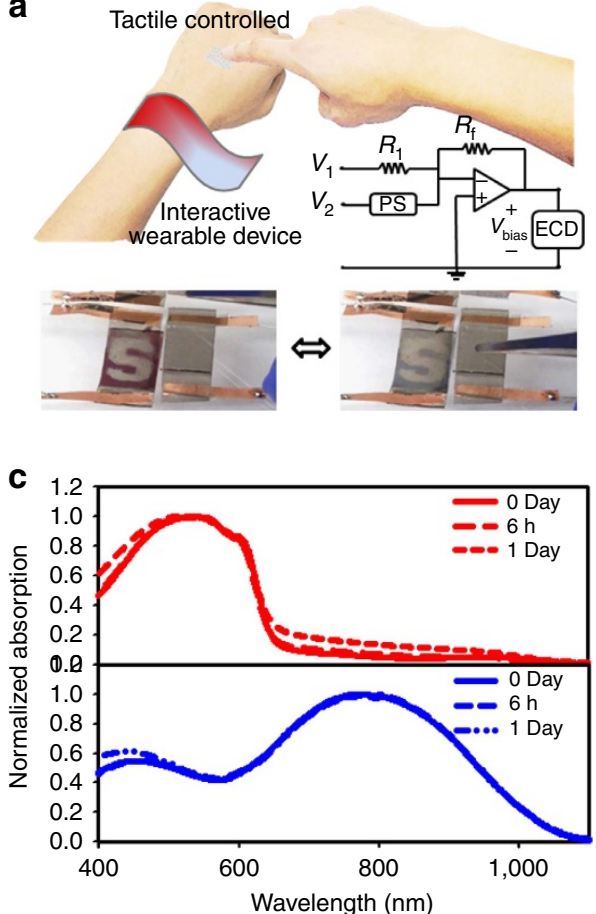

e

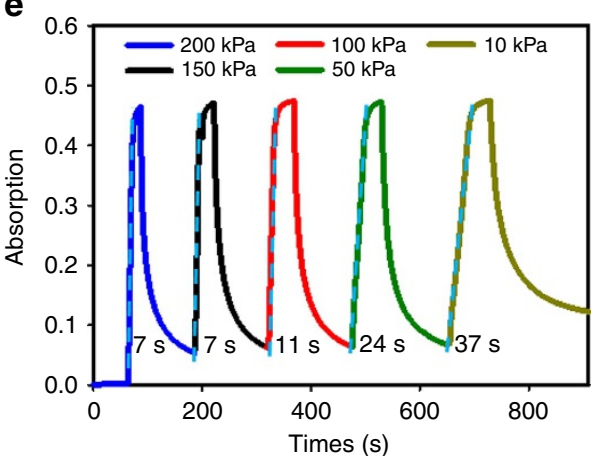

b

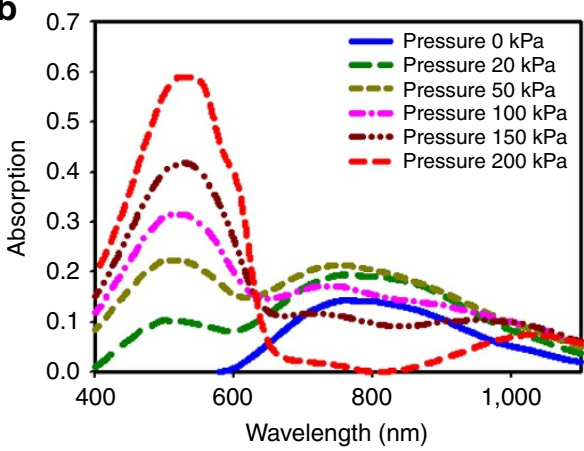

d

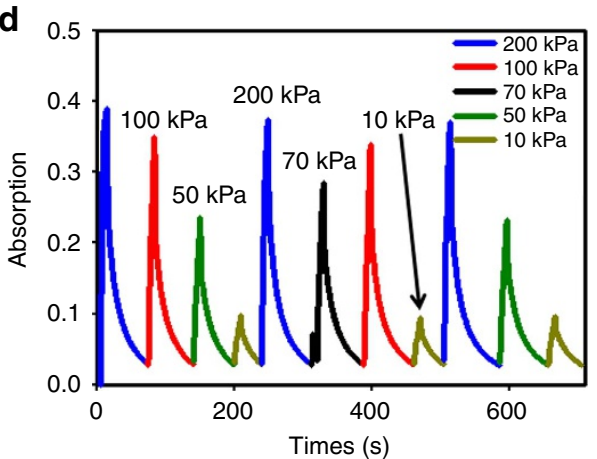

f

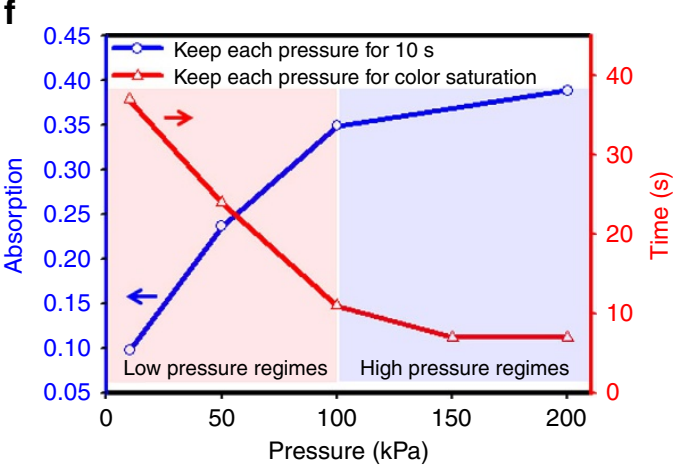

Figure 6 | The schematic layout and measurement of the integrated systems. (a) The schematic layout of interactive colour-changeable e-skin, the layout of the circuit and the photos of PSEC. (b) The absorption spectra related to the pressures. (c) The colour retention properties of ECDs. (d) The realtime absorption changes corresponding to the various applied pressures, in which each pressure was mainatined for $10 \mathrm{~s}$. (e) The speed of colour saturation for different pressures, each pressure was applied till colour saturation for all cases. (f) The absorption and time versus the pressure for both the low- and high-pressure regimes.

correspond to different absorption spectrum/colour. Thus, the real-time absorption change can be used to determine the magnitude of the pressure that was applied. In addition, various pressures were applied to the PSEC system until the colour of the ECDs was saturated (Fig. 6e). The time required to reach colour saturation was found to decrease upon increasing applied pressure. For example, the applied pressure of $200 \mathrm{kPa}$ required $7 \mathrm{~s}$ to achieve colour saturation, whereas an applied pressure of $10 \mathrm{kPa}$ can only reach colour saturation after $37 \mathrm{~s}$. Notably, the value of the slope can be used to quantify the magnitude of pressure we applied. The PSEC system took a longer time to achieve colour saturation compared with our stretchable polymer-based ECDs described in this work. We propose that the slower response is because the pressure-driven voltage has a time delay to reach the proper voltage. The slope of each response curve was decreased when a lower pressure was applied. Importantly, based on the results of the response-time measurement, the lighter pressure regime from 0 to $100 \mathrm{kPa}$ (red region) provides a marked absorption change and saturation time change (Fig. 6f), whereas the higher pressure regime from 100 to $200 \mathrm{kPa}$ (blue region) shows only a slight absorption change and saturation time change. Consequently, the PSEC system can be operated at high pressures to achieve quicker colour saturation. Alternatively, it can be driven at a lower pressure to provide a larger dynamic range to fine-tune the various colours.

As a demonstration of an e-skin with both interactive colourchanging and tactile-sensing properties, a stretchable PS and a stretchable colour-changeability are mounted and connected onto the abdomen and the back of hand of a commercially purchased teddy bear (Fig. 7). Upon applying a weak handshake $(\sim 50 \mathrm{kPa})$, the colour of ECD turned from dark red to blue grey. Releasing the handshake reverts the colour to dark red, whereas applying a strong handshake $(\sim 200 \mathrm{kPa})$ changes the colour again to pale blue. This demonstrates that the feasibility of expressing information from tactile sensing into visible colour changes, and also the tunability of the skin colour related to the various pressures we applied, providing an important function of certain animal skins (Fig. 7 and Supplementary Movie 1). Notably, for the user-interactive devices, the toxicity and carcinogenicity of carbon nanotubes have raised concerns as they have rather 

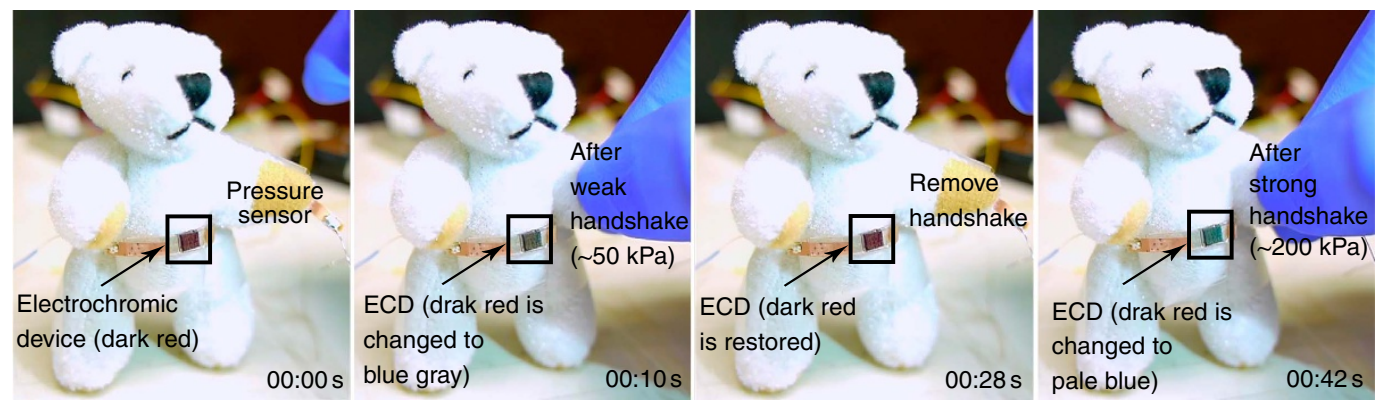

Figure 7 | An interactive colour-changing and tactile-sensing e-skin. Sequential images of a teddy bear show the expression of tactile sensing into visible colour changes. In specific, the original colour of the ECD is changed from dark red to blue grey upon a weak applied pressure $(\sim 50 \mathrm{kPa})$, reverts back to dark red upon pressure release and changes to pale blue upon a strong applied pressure ( $\sim 200 \mathrm{kPa}$; left to right images). The number at the bottom right corner of each image indicates the elapsed time.

similar shapes as asbestos. Previous reports have demonstrated that the longer and thicker carbon nanotubes (lengths $>5 \mu \mathrm{m}$ and diameter $>20 \mathrm{~nm}$ ) will induce significantly more DNA damage and inflammation compared with the lower aspect ratio $^{63,64}$. Here we use the much shorter and smaller diameter SWNT (bundle lengths range from 0.5 to $1.5 \mu \mathrm{m}$, along with an average bundled diameters of $4-5 \mathrm{~nm}$ ), which should greatly reduce the potentially adverse effect. Furthermore, we also consider that proper encapsulation of this system is needed. A number of elastic substrates, such as silicone, polyurethane or fluoroelastomers, are biocompatible and highly stretchable ${ }^{65-67}$. They are also easily processed. Therefore, we believe that encapsulation with such elastomers is a potentially compliant method for further e-skin applications.

\section{Discussion}

In summary, we have successfully demonstrated a chameleoninspired stretchable e-skin capable of interactive colour changes and tactile-sensing properties. This was accomplished via a stretchable, transparent and highly tunable resistive PS. In addition, fully stretchable organic ECDs were fabricated and demonstrated by all-solution processing. Our fabricated PS was observed to demonstrate a wide resistance value range, and, more importantly, the threshold of resistance switching range is tunable by modifying the height of SWNTs on microstructural pyramids. Integration with e-skin rendered this fully stretchable organic ECD electrochromically active. A stretchable colour-changeable e-skin with tactile-sensing control was also demonstrated, in which the applied pressure on the e-skin can be directly expressed through colour changes. Last, we demonstrated that this stretchable colour-changeable e-skin can maintain its skin colour without any applied pressure, indicating its low power consumption. In our future studies, we aim to introduce various colours of electrochromic polymers and array designs to enable a wider and more dynamic colour range for high contrast and high resolution. Such systems should be promising for applications in interactive wearable devices, military applications, artificial prosthetics and smart robots.

\section{Methods}

Characterization. The absorption measurement was carried out by Agilent Cary 6000i UV/Vis/NIR. Scanning electron microscopy (SEM) was performed using an FEI Magellan 400 XHR microscope with a $5-\mathrm{kV}$ accelerating voltage and $25 \mathrm{pA}$ current. The conductivity of the SWNT/PDMS substrate was measured using a standard four-point probe method at room temperature. The resistance was obtained using an Agilent E4980A Precision LCR meter. The stretch property was obtained using the home-made stretchable station. PS cycling measurements were performed on a mechanized $z$ axis stage (Newmark Systems, $0.1 \mathrm{~mm}$ resolution), and a force gauge (Mark 10) was used to apply loads to the $1-\mathrm{cm}^{2}$ pressure- sensitive pad on a custom-built probe station. The load values were recorded by a precision balance.

Preparation of PDMS substrate. A 10:1 mixture of PDMS elastomer base (Sylgard 184, Dow Corning) to curing agent was mixed and stirred for $2 \mathrm{~min}$. The mixture was then evacuated in a vacuum desiccator until the air bubbles were no longer visible. Thereafter, the PDMS mixture was poured into a Petri dish (diameter $=150 \mathrm{~mm}$ ). Finally, the Petri dish containing the PDMS mixture was placed on a hotplate for $8 \mathrm{~h}$ at $60^{\circ} \mathrm{C}$ to cure the PDMS. It is important to have a leveled surface on the hotplate to ensure the PDMS thickness uniformity.

Preparation of pyramidal-microstructured PDMS. A patterned silicon mould (with $34 \mu \mathrm{m}$ height, $10 \times 10 \mu \mathrm{m}^{2}$ top, $50 \times 50 \mu \mathrm{m}^{2}$ base and $41 \mu \mathrm{m}$ periodic spacing pyramidal microstructure) was prepared by fluorination treatment after oxygen plasma for $5 \mathrm{~min}$ at $200 \mathrm{~W}$. A 10:1 mixture of PDMS elastomer base (Sylgard 184, Dow Corning) to curing agent was mixed and for $2 \mathrm{~min}$ and was then evacuated in a vacuumed desiccator until the air bubbles were no longer visible. The PDMS mixture was then poured onto the patterned silicon mould. Finally, the PDMScovered silicon mould was placed on a hotplate for $8 \mathrm{~h}$ at $60^{\circ} \mathrm{C}$ to cure the PDMS.

Preparation of SWNT solution. Arc-discharge SWNT $(2.5 \mathrm{mg})$, purchased from Carbon Solution, Inc (P2-SWNT) was dispersed in NMP ( $N$-methylpyrrolidinone) $(17 \mathrm{ml})$ by sonicating the solution for $30 \mathrm{~min}$ in an ultrasonicator (Cole Parmer) at $30 \%$ amplitude. Subsequently, the solution was centrifuged at 8,000 r.p.m. for $30 \mathrm{~min}$, and the top $80 \%$ supernatant was extracted out for spray coating.

Spray coating of SWNT. The PDMS substrate and pyramidal-microstructured PDMS substrate were first activated with ozone for $20 \mathrm{~min}$ and were then placed on a hotplate at $200^{\circ} \mathrm{C}$. The SWNTs were uniformly spray coated on top of the PDMS surfaces at a distance of $\sim 15 \mathrm{~cm}$ by using an airbrush pressure of 60 p.s.i. until the desired resistance was reached $\left(\sim 2.0{\mathrm{k} \Omega \mathrm{Sq}^{-1}}^{-1}\right.$.

Fabrication of stretchable and ultrathin ECDs. The SWNT/PDMS stack was created by spin coating the P3HT $\left(10 \mathrm{mg} \mathrm{ml}^{-1}\right.$ in chloroform) onto the SWNTs at 1,000 r.p.m. for $1 \mathrm{~min}$ to form a polymer thin film. Thereafter, the gel electrolyte ( $\mathrm{LiClO}_{4}$ :poly(methyl methacrylate): $\mathrm{PC}: \mathrm{CH}_{3} \mathrm{CN}=3: 7: 20: 70$ by weight) was brush coated on top ${ }^{68}$. Finally, the other SWNT-coated PDMS counter electrode was placed on the P3HT-coated SWNT/PDMS substrate to assemble the ECDs. For the ultrathin ECDs, the 1.2- $\mu$ m PEN (Tenji Dupont Film, TeonexFilm Q70) was well attached on a PDMS substrate. The fabrication process of this ultrathin PEN substrate is similar to that of PDMS. The difference between the processing of these two substrates is that we spray-coated SWNT at a lower temperature of $140^{\circ} \mathrm{C}$ on the PEN substrate due to the glass transition temperature of PEN is $155^{\circ} \mathrm{C}$. We used a laser-cut PDMS membrane that had S-shape as a stencil mask to make the patterned ECDs.

Fabrication of resistive PSs. The pyramidal-microstructured PDMS $(34 \mu \mathrm{m}$ height, $10 \times 10 \mu \mathrm{m}^{2}$ top, $50 \times 50 \mu \mathrm{m}^{2}$ base and $41 \mu \mathrm{m}$ periodic spacing) was fabricated using the same method as reported previously and described above ${ }^{6,32}$. A layer of SWNTs was then uniformly spray coated on top of the pyramidalmicrostructured PDMS surface. A kapton tape was applied with different pressures (that is, 1,10 and $30 \mathrm{kPa}$ ) to remove the SWNT from the pyramidal surface, designated as PS-1, PS-10 and PS-30, respectively. Finally, another SWNT/PDMS was used as the counter electrode and was placed on the top of SWNT-coated pyramidal-microstructured PDMS to give the tunable resistive PS. 
PSEC system integration. The PSEC system is fabricated by integrating the stretchable ECD and resistive PS into a summing amplifier circuit. As depicted in Fig. 6a, the summing amplifier applies a bias voltage $\left(V_{\text {bias }}\right)$ across the ECDs as a function of the PS resistance $\left(R_{\mathrm{ps}}\right)$. This behaviour is governed by the following equation:

$$
V_{\text {bias }}=-\left(\frac{V_{1} R_{\mathrm{f}}}{R_{1}}+\frac{V_{2} R_{\mathrm{f}}}{R_{\mathrm{ps}}}\right)
$$

As determined by the bias minimums for switching between neutral and oxidized states $V_{1}$ and $V_{2}$ were, respectively, set to -1 and $1 \mathrm{~V}$. Both $R_{1}$ and $R_{\mathrm{f}}$ are diodes with a resistance of $30 \mathrm{k} \Omega$, and $R_{\mathrm{f}}$ a diode resistance of $30 \mathrm{k} \Omega$. $R_{\mathrm{ps}}$ is the resistance of the PS. Without pressure, $R_{\mathrm{ps}}$ is several orders of magnitude larger than $R_{\mathrm{f}}$, resulting in a bias voltage of $-1 \mathrm{~V}$. As pressure is applied, $R_{\mathrm{ps}}$ decreases, and $V_{\text {bias }}$ approaches $+1 \mathrm{~V}$.

Measurement of the absorption spectrum versus applied pressure. The PSEC system was integrated with the stretchable ECD and resistive PS as described above. To characterize the impact of applied pressure on the real-time absorption change, the ECD was attached on the thin-film stage of Agilent Cary 6000i UV/Vis/NIR. As different pressures were applied on the PS, the colour change of the ECD can be measured by the Agilent Cary 6000i UV/Vis/NIR, and the applied pressure on the PS was determined by a balance simultaneously.

\section{References}

1. Hammock, M. L., Chortos, A., Tee, B. C.-K., Tok, J. B.-H. \& Bao, Z. 25th anniversary article: the evolution of electronic skin (e-skin): a brief history, design considerations, and recent progress. Adv. Mater. 25, 5997-6038 (2013).

2. Dahiya, R. S., Metta, G., Valle, M. \& Sandini, G. Tactile sensing-from humans to humanoids. IEEE Trans. Robotics 26, 1-20 (2010).

3. Dahiya, R. S., Mittendorfer, P., Valle, M., Cheng, G. \& Lumelsky, V. Directions towards effective utilization of tactile skin-a review. IEEE Sensors J. 13, 4121-4138 (2013).

4. Kim, D. H. et al. Epidermal electronics. Science 333, 838-843 (2011).

5. Takei, K. et al. Nanowire active-matrix circuitry for low-voltage macroscale artificial skin. Nat. Mater. 9, 821-826 (2010).

6. Mannsfeld, S. C. B. et al. Highly sensitive flexible pressure sensors with microstructured rubber dielectric layers. Nat. Mater. 9, 859-864 (2010).

7. Lipomi, Darren J. et al. Skin-like pressure and strain sensors based on transparent elastic films of carbon nanotubes. Nat. Nanotechnol. 6, 788-792 (2011)

8. Lipomi, D. J., Tee, B. C.-K., Vosgueritchian, M. \& Bao, Z. Stretchable organic solar cells. Adv. Mater. 23, 1771-1775 (2011).

9. Kaltenbrunner, M. et al. An ultra-lightweight design for imperceptible plastic electronics. Nature 499, 458-463 (2013).

10. Tee, B. C.-K., Wang, C., Allen, R. \& Bao, Z. An electrically and mechanically self-healing composite with pressure- and flexion-sensitive properties for electronic skin applications. Nat. Nanotechnol. 7, 825-832 (2012).

11. White, M. S. et al. Ultrathin, highly flexible and stretchable PLEDs. Nat. Photon. 7, 811-816 (2013)

12. Liang, J., Li, L., Niu, X., Yu, Z. \& Pei, Q. Elastomeric polymer light-emitting devices and displays. Nat. Photon. 7, 817-824 (2013).

13. Wang, C. et al. User-interactive electronic skin for instantaneous pressure visualization. Nat. Mater. 21, 899-904 (2013).

14. Lumelsky, V. J., Shur, M. S. \& Wagner, S. Sensitive skin. IEEE Sens. J. 1, 41-51 (2001).

15. Kim, R.-H. et al. Waterproof AlInGaP optoelectronics on stretchable substrates with applications in biomedicine and robotics. Nat. Mater. 9, 929-937 (2010).

16. Someya, T. et al. A large-area, flexible pressure sensor matrix with organic field effect transistors for artificial skin applications. Proc. Natl Acad. Sci. USA 101, 9966-9970 (2004)

17. Schwartz, G. et al. Flexible polymer transistors with high pressure sensitivity for application in electronic skin and health monitoring. Nat. Commun. 4, 1859 (2013).

18. Yu, Z., Niu, X., Liu, Z. \& Pei, Q. Intrinsically stretchable polymer light-emitting devices using carbon nanotube-polymer composite electrodes. Adv. Mater. 23, 3989-3994 (2011).

19. Gerratt, A. P., Michaud, H. O. \& Lacour, S. P. Elastomeric electronic skin for prosthetic tactile sensation. Adv. Funct. Mater. 25, 2287-2295 (2015).

20. Gerratt, A. P., Sommer, N., Lacour, S. P. \& Billard, A.14th IEEE-RAS International Conference. Stretchable capacitive tactile skin on humanoid robot fingers-first experiments and results. Humanoid Robots (Humanoids) 238-245 (2014).

21. Graz, I. et al. Flexible active-matrix cells with selectively poled bifunctional polymer-ceramic nanocomposite for pressure and temperature sensing skin. J. Appl. Phys. 106, 034503 (2009).

22. Michaud, H. O., Teixidor, J. \& Lacour, S. P. 28th IEEE International Conference. Soft flexion sensors integrating strechable metal conductors on a silicone substrate for smart glove applications. Micro Electro Mech. Syst. (MEMS). pp 760 (2015)
23. Kim, J. et al. Stretchable silicon nanoribbon electronics for skin prosthesis. Nat. Commun. 5, 5747 (2014)

24. Son, D. et al. Multifunctional wearable devices for diagnosis and therapy of movement disorders. Nat. Nanotechnol. 9, 397-404 (2014).

25. Jung, S., Lee, J., Hyeon, T., Lee, M. \& Kim, D.-H. Fabric-based integrated energy devices for wearable activity monitors. Adv. Mater. 26, 6329-6334 (2014).

26. Teyssier, J., Saenko, S. V., van der Marel, D. \& Milinkovitch, M. C. Photonic crystals casue active colour change in chameleons. Nat. Commun. 6, 6368 (2015).

27. Bauer, S. et al. 25th anniversary article: a soft future: from robots and sensor skin to energy harvesters. Adv. Mater. 26, 149-162 (2014).

28. Haque, Md. A., Kurokawa, T., Kamita, G., Yue, Y. \& Gong, J. P. Rapid and reversible tuning of structural colour of a hydrogel over the entire visible spectrum by mechanical stimulation. Chem. Mater. 23, 5200-5207 (2011).

29. Zhao, Q. et al. Electric-field-tuned colour in photonic crystal elastomers. Appl. Phys. Lett. 100, 101902 (2012).

30. van den Ende, D., Kamminga, J.-D., Boersma, A., Andritsch, T. \& Steeneken, P. G. Voltage-controlled surface wrinkling of elastomeric coatings. Adv. Mater. 25, 3438-3442 (2013)

31. Morin, S. A. et al. Camouflage and display for soft machines. Science 337, 828 (2012)

32. Yu, C. et al. Adaptive optoelectronic camouflage systems with designs inspired by cephalopod skins. Proc. Natl Acad. Sci. USA 111, 12998-13003 (2014).

33. Wang, Q., Gossweiler, G. R., Craig, S. L. \& Zhao, X. Cephalopod-inspired design of electro-mechano-chemically responsive elastomers for on-demand fluorescent patterning. Nat. Commun. 5, 4899 (2014).

34. Wu, W., Wen, X. \& Wang, Z. L. Taxel-addressable matrix of vertical-nanowir piezotronic transistors for active and adaptive tactile imaging. Science 340, 952-957 (2013).

35. Fan, Z. et al. Toward the development of printable nanowire electronics and sensors. Adv. Mater. 21, 3730-3743 (2009).

36. Metzger, C. et al. Flexible-foam-based capacitive sensor arrays for object detection at low cost. Appl. Phys. Lett. 92, 013506 (2008).

37. Graz, I. et al. Flexible ferroelectret field-effect transistor for large-area sensor skins and microphones. Appl. Phys. Lett. 89, 073501 (2006).

38. Takahashi, T., Takei, K., Gillies, A. G., Fearing, R. S. \& Javey, A. Carbon nanotube active-matrix backplanes for conformal electronics and sensors. Nano Lett. 11, 5408-5413 (2011)

39. Pang, C. et al. A flexible and highly sensitive strain-gauge sensor using reversible interlocking of nanofibres. Nat. Mater. 11, 795-801 (2012).

40. Yamada, T. et al. A stretchable carbon nanotube strain sensor for human motion detection. Nat. Nanotechnol. 6, 296-301 (2011).

41. Tee, B. C.-K. et al. Tunable flexible pressure sensors using microstructured elastomer geometries for intuitive electronics. Adv. Funct. Mater. 24, 5427-5434 (2014).

42. Choong, C.-L. et al. Highly stretchable resistive pressure sensors using a conductive elastomeric composite on a micropyramid array. Adv. Mater. 26, 3451-3458 (2014).

43. Wang, X., Gu, Y., Xiong, Z., Cui, Z. \& Zhang, T. Silk-molded flexible, ultrasensitive, and highly stable electronic skin for monitoring human physiological signals. Adv. Mater. 26, 1336-1342 (2014).

44. Somani, P. R. \& Radhakrishnan, S. Electrochromic materials and devices: present and future. Mater. Chem. Phys. 77, 117-133 (2002).

45. Ma, D., Shi, G., Wang, H., Zhang, Q. \& Li, Y. Hierarchical NiO microflake films with high colouration efficiency, cyclic stability and low power consumption for applications in a complementary electrochromic device. Nanoscale 5, 4808-4815 (2013).

46. Deutschmann, T. \& Oesterschulze, E. Integrated electrochromic iris device for low power and space-limited applications. J. Opt. 16, 075301 (2014).

47. Yan, C. et al. Stretchable and wearable electrochromic devices. ACS Nano 8, 316-322 (2014)

48. Olivier, Y. et al. 25th anniversary article: high-mobility hole and electron transport conjugated polymers: how structure defines function. Adv. Mater. 26, 2119-2136 (2014).

49. Kerszulis, J. A., Amb, C. M., Dyer, A. L. \& Reynolds, J. R. Follow the yellow brick road: structural optimization of vibrant yellow-to-transmissive electrochromic conjugated polymers. Macromolecules 47, 5462-5469 (2014).

50. Savagatrup, S., Printz, A. D., O’Connor, T. F., Zaretski, A. V. \& Lipomi, D. J. Molecularly stretchable electronics. Chem. Mater. 26, 3028-3041 (2014).

51. Österholm, A. M. et al. Four shades of brown: tuning of electrochromic polymer blends toward high-contrast eyewear. ACS Appl. Mater. Interfaces 7, 1413-1421 (2015).

52. Ng, T. N. et al. Scalable printed electronics: an organic decoder addressing ferroelectric non-volatile memory. Sci. Rep. 2, 585 (2012).

53. Thakur, V. K., Ding, G., Ma, J., Lee, P. S. \& Lu, X. Hybrid materials and polymer electrolytes for electrochromic device applications. Adv. Mater. 24, 4071-4096 (2012). 
54. Mortimer, R. J. Electrochromic materials. Annu. Rev. Mater. Res. 41, 241-268 (2011).

55. Öktem, G., Balan, A., Barana, D. \& Toppare, L. Donor-acceptor type random copolymers for full visible light absorption. Chem. Commun. 47, 3933-3935 (2011).

56. Beverina, L., Pagani, G. A. \& Sassi, M. Multichromophoric electrochromic polymers: colour tuning of conjugated polymers through the side chain functionalization approach. Chem. Commun. 50, 5413-5430 (2014).

57. Zhu, Y. et al. Neutral colour tuning of polymer electrochromic devices using an organic dye. Chem. Commun. 50, 8167-8170 (2014).

58. Argun, A. A. et al. Multicoloured electrochromism in polymers: structures and devices. Chem. Mater. 16, 4401-4412 (2004).

59. O'Connor et al. Correlations between mechanical and electrical properties of polythiophenes. ACS Nano 4, 7538-7544 (2010).

60. O'Connor et al. Anisotropic structure and charge transport in highly strainaligned regioregular poly(3-hexylthiophene). Adv. Funct. Mater. 21, 3697-3705 (2011).

61. Gargi, D. et al. Charge transport in highly face-on poly(3-hexylthiophene) films. J. Phys. Chem. C 117, 17421-17428 (2013).

62. $\mathrm{Wu}, \mathrm{H}$--C. et al. A rapid and facile soft contact lamination method: evaluation of polymer semiconductors for stretchable transistors. Chem. Mater. 26, 4544-4551 (2014)

63. Kostarelosl, K. The long and short of carbon nanotube toxicity. Nat. Biotechnol. 26, 774-776 (2008).

64. Yamashita, K. et al. Carbon nanotubes elicit DNA damage and inflammatory response relative to their size and shape. Inflammation 33, 276-280 (2010).

65. Belanger, M.-C. \& Marois, Y. Hemocompatibility, biocompatibility, inflammatory and in vivo studies of primary reference materials low-density polyethylene and polydimethylsiloxane: a review. J. Biomed. Mater. Res. 58, 467-477 (2001)

66. Axisa, F. et al. Design and fabrication of a low cost implantable bladder pressure monitor. Proc. IEEE Eng. Med. Biol. Soc. 31, 4864-4867 (2009).

67. May, P. et al. Phosphonitrilic fluoroelastomer lined denture. US Patent 4,251,215 (1981)

68. Argun, A. A., Cripan, A. \& Reynolds, J. R. The first truly all-polymer electrochromic devices. Adv. Mater. 15, 1338-1341 (2003).

\section{Acknowledgements}

H.-H.C. acknowledges supports from the Ministry of Science and Technology of the Republic of China. We gratefully acknowledge support from the MSIP (Ministry of Science, ICT and Future Planning), Korea, under the IT Consilience Creativity Program (NIPA-2014-H0201-14-1001) supervised by the NIPA (National IT Industry Promotion Agency).

\section{Author contributions}

H.-H.C., A.N., A.C., J.M. and Z.B. conceived the concept, processing and structure details. A.N., J.W.F.T. and C.L. assisted with device fabrication and characterization. T.K. prepared and purified the materials. H.-H.C. and W.-G.B. prepared the figures. H.-H.C., J.B.-H.T and Z.B. co-wrote the paper. All authors discussed the results and commented on the manuscript.

\section{Additional information}

Supplementary information accompanies this paper at http://www.nature.com/ naturecommunications

Competing financial interests: The authors declare no competing financial interests.

Reprints and permission information is available online at http://npg.nature.com/ reprintsandpermissions/

How to cite this article: Chou, H.-H. et al. A chameleon-inspired stretchable electronic skin with interactive colour changing controlled by tactile sensing. Nat. Commun. 6:8011 doi: $10.1038 /$ ncomms9011 (2015).

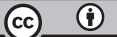

This work is licensed under a Creative Commons Attribution 4.0 International License. The images or other third party material in this article are included in the article's Creative Commons license, unless indicated otherwise in the credit line; if the material is not included under the Creative Commons license, users will need to obtain permission from the license holder to reproduce the material. To view a copy of this license, visit http://creativecommons.org/licenses/by/4.0/ 